Pacific Journal of

Mathematics

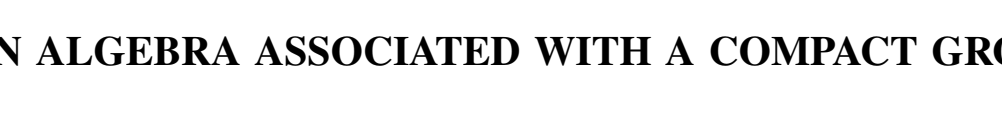




\section{AN ALGEBRA ASSOCIATED WITH A COMPACT GROUP}

\section{MEYER JERISON}

1. Introduction. This paper deals with a variation on a familiar theme; namely, a proof that a space is determined, in some appropriate sense, by certain properties of a collection of functions on that space. Here, the space in question is a compact abelian group $G$, and the collection of functions is the set of all continuous functions from $G$ into a commutative, complex Banach algebra $R$. The relevant properties of the collection of functions make it into a Banach algebra $R(G)$, with addition as well as multiplication by scalars defined in the usual way, that is, pointwise, norm defined by

$$
\|x\|=\sup _{g \in G}\|x(g)\| \quad x \in R(G),
$$

and multiplication of elements in $R(G)$ defined as the convolution

$$
(x y)(g)=\int_{G} x\left(g h^{-1}\right) y(h) d h .
$$

The integral, like all integrals appearing in this paper, is taken with respect to Haar measure in $G$, normalized so that the measure of $G$ is 1. The integrand takes on values in the Banach algebra $R$, and the integral is of the type described in [3]. An alternate approach to this integral is obtained by observing that, as continuous functions on a compact group, the functions with which we deal are almost periodic in the sense of [2], and the integral is the invariant mean whose existence and uniqueness are proved in [2].

We will let $\mathscr{H}$ denote the class of theorems of the type described in the first sentence of the preceding paragraph. Many theorems of this class may be found in the literature; the ones most intimately related to the present investigation appear in [6] and the papers quoted there. I feel, therefore, that some justification is needed for the publication of still another one. Futhermore, there is probably no limit to the number of different kinds of spaces and different sets of functions which might be combined to yield a theorem in $\mathscr{H}$. The choice of the particular set-up that is being studied here was motivated by an attempt to solve a problem in topology proposed by Fox [4].

If $X, Y$, and $Z$ are topological spaces, and if the cartesian product $X \times Y$ is homeomorphic with $X \times Z$, then it is known that $Y$ and $Z$ need not be homeomorphic. In the simplest example of this phenomenon [4], $Y$ and $Z$ are compact subsets of the plane that are not at all

Received June 15, 1954. 
pathological, and $X$ is a closed interval. The non-homogeneity of $X$ at the end points seems to be what makes this example work, and Fox raises the question whether homeomorphism of the products will imply homeomorphism of $Y$ and $Z$ if $X$ is a circle. Now, let $R_{1}$ and $R_{2}$ be the Banach algebras of all continuous complex (real would work just as well) valued functions on the compact spaces $Y$ and $Z$, respectively, with multiplication as well as addition defined pointwise. Then a well known theorem (in $\mathscr{H}$ ) asserts that $R_{1}$ and $R_{2}$ are isomorphic, in symbols ${ }^{1} R_{1} \approx R_{2}$, if, and only if, $Y$ and $Z$ are homeomorphic. A function from $X$ into $R_{1}$ may be identified in an obvious way with a complex function on $X \times Y$. If one could prove that isomorphism of the algebra of all continuous functions from the circle $X$ into $R_{1}$ with the space of functions from $X$ into $R_{2}$ implies $R_{1} \approx R_{2}$, and if, moreover, the former isomorphism is a consequence of the homeomorphism of $X \times Y$ with $X \times Z$, then Fox's problem would be solved.

It would be pleasant to be able to report that this has been achieved, especially because the theorems in $\mathscr{H}$ have had no noteworthy applications to problems in topology. Unfortunately, although Theorem 2 does say that $R_{1}(G) \approx R_{2}(G)$ implies $R_{1} \approx R_{2}$ for any compact abelian group $G$, in particular for a circle, the algebraic structure which has been placed on $R(G)$ is of such a nature that I cannot prove that homeomorphism of $G \times Y$ with $G \times Z$ implies $R_{1}(G) \approx R_{2}(G)$. Section 4 is devoted to a discussion of some of the reasons for the failure of this approach.

Theorem 2 is probably true without the hypothesis that $G$ is abelian, but I have not been able to prove it. This hypothesis does not influence the applicability of the theorem to Fox's problem. The requirement that $R$ be a commutative Banach algebra whose only idempotent is its unit, is equivalent, if $R$ is the algebra of continuous functions on $Y$ with pointwise multiplication, to the assumption that $Y$ is connected.

2. Complex valued functions. In this section, we assume that $R$ is the field of complex numbers, and then we no longer need to require that $G$ be abelian.

THEOREm 1. If $D$ and $\Delta$ are the Banach algebras of continuous complex functions on the compact groups $G$ and $\Gamma$, respectively (with multiplicatian defined by (2)), and $D \approx \Delta$, then $G$ and $\Gamma$ are isomorphic.

This theorem can probably be proved by the technique of [6], but

1 This symbol will be reserved for isomorphism (including preservation of norms) of Banach algebras. 
we choose to base our proof on the theory of Banach spaces of continuous functions. If $f: \Delta \rightarrow D$ is the isomorphism, then there exists a one-to-one mapping $\varphi$ of $G$ onto $\Gamma$ which is a homeomorphism of the underlying topological spaces, and $x_{0} \in D$ with the property $\left|x_{0}(g)\right| \equiv 1$, such that"

$$
f \xi(g)=x_{0}(g) \cdot \xi(\varphi g)
$$

for all $\xi \in \Delta$ and $g \in G$. We propose to show that $\varphi$ is an isomorphism of the groups.

Lemma $1 . x_{0}\left(g^{\prime} g^{\prime \prime}\right)=x_{0}\left(g^{\prime}\right) x_{0}\left(g^{\prime \prime}\right)$ for all $g^{\prime}, g^{\prime \prime} \in G$.

Proof. If $\xi_{0} \in \Delta$ is defined as $\xi_{0}(\gamma) \equiv 1, \gamma \in \Gamma$, then $f \xi_{0}=x_{0} . \quad$ Consequently,

$$
x_{0} \cdot x_{0}=f \xi_{0} \cdot f \xi_{0}=f\left(\xi_{0} \cdot \xi_{0}\right)=f\left(\xi_{0}\right)=x_{0} ;
$$

that is, $x_{0}$ is an idempotent in $D$. Hence,

$$
x_{0}(g)=x_{0} x_{0}(g)==\int x_{0}\left(g h^{-1}\right) x_{0}(h) d h .
$$

Since $\left|x_{0}(g)\right| \equiv 1$, we have

$$
1=x_{0}(g) x_{0}(g)=\int \overline{x_{0}(g)} x_{0}\left(g h^{-1}\right) x_{0}(h) d h .
$$

But the absolute value of the integrand is 1 for all $g$ and $h$, and the measure of $G$ is also 1 , so that

$$
x_{0}(g) x_{0}\left(g h^{-1}\right) x_{0}(h)=1
$$

for all $g$ and $h$ (more precisely, for almost all $h$, but the function is continuous). Setting $g=g^{\prime} g^{\prime \prime}$ and $h=g^{\prime \prime}$, and remembering that $\left|x_{0}(g)\right|=$ 1 , we obtain $x_{0}\left(g^{\prime} g^{\prime \prime}\right)=x_{0}\left(g^{\prime}\right) \cdot x_{0}\left(g^{\prime \prime}\right)$.

Proof of Theorem 1. It is required only to prove that

$$
\varphi\left(g g^{\prime}\right)=(\varphi g)\left(\varphi g^{\prime}\right) \quad \text { for all } g, g^{\prime} \in G \text {. }
$$

Let $\Omega$ be a neighborhood of the identity in $\Gamma$, and let $\omega$ be a continuous function on $\Gamma$ which vanishes outside of $\Omega$ and such that

$$
\int_{\Gamma} \omega\left(\eta^{-1}\right) \omega(\eta) d \eta=1
$$

${ }^{2}$ A proof of this assertion for a Banach space of real functions may be found in a number of different places, including [1, p. 172]. A generalization which includes the case of complex functions appears in [5, Theorem 6.2]. 
Set

$$
\omega_{1}(\gamma)=\omega\left((\varphi g)^{-1} \gamma\right) \quad \text { and } \quad \omega_{2}(\gamma)=\omega\left(\gamma \cdot\left(\varphi g^{\prime}\right)^{-1}\right) ;
$$

then a straightforward computation yields

$$
\omega_{1} \omega_{2}\left((\varphi g)\left(\varphi g^{\prime}\right)\right)=1 .
$$

Using (2), (3), and Lemma 1 in the relation $f\left(\omega_{1} \omega_{2}\right)=\left(f \omega_{1}\right) \cdot\left(f \omega_{2}\right)$, we find that for any $a \in G$,

$$
\begin{aligned}
f\left(\omega_{1} \omega_{2}\right)(a) & =\int_{G} x_{0}\left(a h^{-1}\right) \cdot \omega_{1}\left(\varphi\left(a h^{-1}\right)\right) \cdot x_{0}(h) \cdot \omega_{2}(\varphi h) d h \\
& =x_{0}(a) \int_{G} \omega\left((\varphi g)^{-1} \varphi\left(a h^{-1}\right)\right) \cdot \omega\left((\varphi h)\left(\varphi g^{\prime}\right)^{-1}\right) d h .
\end{aligned}
$$

Since $\omega$ vanishes outside of $\Omega$, this implies

$$
f\left(\omega_{1} \omega_{2}\right)(\alpha)=0
$$

for $a \notin V$

where

$$
V=\varphi^{-1}[(\varphi g) \Omega] \cdot \varphi^{-1}\left[\Omega\left(\varphi g^{\prime}\right)\right] .
$$

$(\varphi g) \Omega$ is a neighborhood of $\varphi g$ in $I^{\prime}$, so that $\varphi^{-1}[(\varphi g) \Omega]$ is a neighborhood of $g$ in $G$. Similarly, $\varphi^{-1}\left[\Omega\left(\varphi g^{\prime}\right)\right]$ is a neighborhood of $g^{\prime}$, and $V$ is a neighborhood of $g g^{\prime}$.

Let $a$ be the (unique) element of $G$ such that $\varphi a=(\varphi g) \cdot\left(\varphi g^{\prime}\right)$, and suppose $a \neq g g^{\prime}$. Since no previous restrictions have been placed upon $\Omega$, we may now choose $\Omega$ so that $a \notin V$, that is, so that $f\left(\omega_{1} \omega_{2}\right)(a)=0$. But,

$$
f\left(\omega_{1} \omega_{2}\right)(a)=x_{0}(\alpha) \cdot \omega_{1} \omega_{2}(\varphi a)=x_{0}(\alpha) \cdot \omega_{1} \omega_{2}\left(\varphi g \cdot \varphi g^{\prime}\right)=x_{0}(a) \neq 0 .
$$

This contradicts the assumption that $a \neq g g^{\prime}$, and therefore $\phi\left(g g^{\prime}\right)=$ $(\varphi g)\left(\varphi g^{\prime}\right)$.

\section{The isomorphism theorem.}

THEOREM 2. Let $G_{1}$ and $G_{2}$ be compact abelian groups, and $R_{1}$ and $R_{2}$, commutative Banach algebras whose only idempotents are their respective units. Then $R_{1}\left(G_{1}\right) \approx R_{2}\left(G_{2}\right)$ if, and only if, $R_{1} \approx R_{2}$ and $G_{1}$ is isomorphic to $G_{\text {.. }}$.

Proof. In one direction, the implication is trivial. To prove the non-trivial half of the theorem, we consider a group $G$, an algebra $R$ with unit $e$, and show how $R$ and $G$ may be recovered from $R(G)$, using only the structure of $R(G)$ as a Banach algebra.

The first step is to find $D$ (in the natation of Theorem 1 ) in $R(G)$. 
Specifically, we want to characterize the set $D e$ of elements in $R(G)$ of the form $\lambda(g) \cdot e$, where $\lambda(g)$ is a complex function on $G$.

Lemma 2. De is the smallest closed linear subspace containing all of the idempotents of $R(G)$.

Proof. We review some essential facts concerning Fourier analysis in $R(G)$; the proofs may be found in [2]. Let $\left\{\chi_{a}\right\}$ be the set of all continuous characters of $G$, that is,

$$
\left|\chi_{\alpha}(g)\right|=1 \text { and } \chi_{\alpha}\left(g g^{\prime}\right)=\chi_{\alpha}(g) \cdot \chi_{\alpha}\left(g^{\prime}\right)
$$

for all $g, g^{\prime} \in G$. For $x \in R(G)$, define

$$
r_{\alpha}=\int_{G} \overline{\chi_{\alpha}}(g) x(g) d g \text {. }
$$

This is an element of $R$. The formal series $\sum_{\alpha} \chi_{\alpha}(g) r_{\alpha}$ represents $x(g)$ in exactly the same way that classical Fourier series represent continuous functions. We write $x \sim \sum \chi_{\alpha} r_{\alpha}$. If $x^{\prime} \sim \sum \chi_{\alpha} r_{\alpha}^{\prime}$ then $x x^{\prime} \sim \sum \chi_{\alpha} r_{\alpha} r_{\alpha}^{\prime}$. (This is not proved in [2], but can be done, as in the classical case, simply by evaluating the $\alpha$ th coefficient of $x x^{\prime}$.)

Since the formal series representation is unique, $x$ is an idempotent if, and only if, $r_{\alpha}=0$ or $e$ for all $\alpha$. Thus, every idempotent of $R(G)$ is in $D e$, and, in fact, is an idempotent of $D$ multiplied by $e$. Since the idempotents of $D$ span $D$, the idempotents of $R(G)$ span $D e$. It is obvious, that $D e$ is a closed linear subspace of $R(G)$.

Lemma 2 asserts that $D e$ is determined by $R(G)$. Since $D e \approx D$ (assuming $\|e\|=1$ ), it follows from Theorem 1 that $G$ is determined by $R(G)$. It remains only to prove that $R$ is determined by $R(G)$, and this will be achieved essentially by fishing the constant functions out of $R(G)$. Specifically, we will find all of the constant functions multiplied by some character of $G$. It is impossible to distinguish between characters using only their algebraic properties in $R(G)$.

LEMMA 3. Let $x$ be any irreducible idempotent of $R(G)$, that is, any idempotent which is not the sum of other non-zero idempotents. The principal ideal generated by $x$ is isomorphic with the Banach algebra $R$.

Proof. From the discussion of idempotents given earlier, it is clear that $x=\chi e$ for some character $\chi$ of $G$. If $y \in R(G)$, then

$$
\begin{aligned}
y x(g) & =\int y\left(g h^{-1}\right) \chi(h) \cdot e d h=\int y(k) \chi\left(k^{-1} g\right) \cdot e d k \\
& =\left[\int y(k) \bar{\chi}(\bar{k}) d k\right] \chi(g) \cdot e=\chi(g) \cdot r
\end{aligned}
$$


where $r$ is the "Fourier coefficient" of $y$ with respect to the character $\chi$. Similarly $x y(g)=\chi(g) r$. Consequently, the set of functions in $R(G)$ of the form $\chi(g) r, r \in R$, is a two-sided ideal. The correspondence $r \leftrightarrow \chi r$ is the desired isomorphism.

4. Fox's problem. It was remarked earlier that the class of theorems $\mathscr{H}$ has been disappointing as a source of solutions of problems in topology, problems that do not involve the function space directly. The comments which will be made here refer only to the failure to solve Fox's problem, but it seems to me that they lie close to the heart of the difficulties in general.

It is unlikely that Theorem 2 can be used to prove Fox's conjecture because the conclusion of the theorem is so strong. What is needed is a theorem with the statement " $G_{1}$ isomorphic to $G_{2}$ ", in the hypothesis rather than in the conclusion. That so much could be proved from the hypothesis $R_{1}\left(G_{1}\right) \approx R_{2}\left(G_{2}\right)$ implies that it is a very strong condition and one that will be difficult to verify. Thus, in the application to Fox's problem, we would take $R_{1}$ and $R_{2}$ as the algebras of continuous functions on $Y$ and $Z$, respectively, (pointwise multiplication) and we would have to prove that if $G \times Y$ is homeomorphic with $G \times Z$, then $R_{1}(G) \approx$ $R_{2}(G)$. One may observe, incidentally, that in the correspondence between these two algebras induced by the homeomorphism of the product spaces, norms are preserved, but the norms do not enter in an essential way into the proof of $R_{1} \approx R_{2}$.

Apparently, then, the source of the difficulty is the peculiar definition of multiplication in $R(G)$. I believe, however, that the trouble goes deeper. A theorem in $\mathscr{H}$ generally has a hypothesis which is so strong that to verify it is tantamount to exhibiting a homeomorphism of the topological spaces on which the functions are defined. One manifestation of this is the fact that the hypothesis implies not only a homeomorphism but also an intimate relationship between the homeomorphism of the conclusion and the isomorphism of the hypothesis, as given by formula (3). The presence of such a formula is implicit in all of the techniques for proving theorems in $\mathscr{H}$. It is what requires the strong hypothesis, which, in turn, limits the applicability of the theorem.

\section{REFERENCES}

1. S. Banach, Théorie des opérations linéaires, Warsaw, 1932.

2. S. Bochner and J. von Neumann, Almost periodic functions in groups II, Trans. Amer. Math. Soc., 37 (1935), 21-50.

3. N. Dunford and B. J. Pettis, Linear operations on summable functions, Trans. Amer. Math. Soc., 47 (1940), 323-392. 
4. R. H. Fox, On a problem of S. Ulam concerning cartesian products, Fund. Math., 34 (1947), 278-287.

5. M. Jerison, The space of bounded maps into a Banach space, Ann. of Math., 52 (1950), 309-327.

6. J. G. Wendel, Left centralizers and isomorphisms of group algebras, Pacific J. Math., 2 (1952), 257-261.

PuRdue UNIVERsity 

.././. ./FrontMatter/paper .pdf 


\section{Pacific Journal of Mathematics}

Nesmith Cornett Ankeny and Theodore Joseph Rivlin, On a theorem of S.

Bernstei........................................ 849

Louis Auslander, The use of forms in variational calculation .......... 853

Paul Civin, Abstract Riemann sum . .......................... 861

Paul Civin, Some ergodic theorems involving two operator ............ 869

Eckford Cohen, The number of solutions of certain cubic congruence .... . 877

Richard M. Cohn, Specializations over difference field .............. 887

Jean Dieudonné, Pseudo-discriminant and Dickson invarian . . ......... 907

Ky Fan, A comparison theorem for eigenvalues of normal matrice ........ 911

Richard P. Gosselin, On the convergence behaviour of trigonometric interpolating polynomial ........................... 915

Peter K. Henrici, On generating functions of the Jacobi polynomial . . . . . . . 923

Meyer Jerison, An algebra associated with a compact grou ............ 933

Wilhelm Magnus, Infinite determinants associated with Hill's equatio . . . . . 941

G. Power and D. L. Scott-Hutton, The slow steady motion of liquid past a semi-elliptical bos................................. 953

Lyle E. Pursell, An algebraic characterization of fixed ideals in certain function ring .................................... 963

C. T. Rajagopal, Additional note on some Tauberian theorems of O. Szás . . 971 Louis Baker Rall, Error bounds for iterative solutions of Fredholm integral

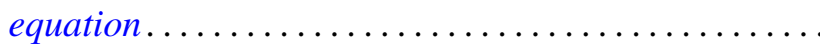

Shigeo Sasaki and Kentaro Yano, Pseudo-analytic vectors on

pseudo-Kählerian manifold ......................

Eugene Schenkman, On the tower theorem for finite group

P. Stein and John E. L. Peck, On the numerical solution of Poisson's equation over a rectangl ........................

Morgan Ward, The mappings of the positive integers into themselves which preserve divisio .

Seth Warner, Weak locally multiplicatively-convex algebra 1025

Louis Weisner, Group-theoretic origin of certain generating function .... 